\title{
Investigação ambulatorial na amenorréia primária: estudo de caso com pseudo isodicêntrico cromossomo $X$
}

\author{
Ambulatory investigation on primary amenorrhea: a pseudo isodicentric $X$ chromosome \\ case study
}

\author{
Investigación ambulatoria en amenorrea primaria: estudio de caso con pseudo \\ isodicéntrico cromosoma $X$
}

Andréa de Oliveira Cecchi ${ }^{1}$, Barbara Mendes Kirchhoff ${ }^{1}$, Camila Graciano Gerotto ${ }^{1}$, Elisabete Lilian Dair $^{1,2}$, Matheus Thiago Alves Martins ${ }^{1}$, Nathália Barbosa Furlan², Ronaldo de Castro Tavares ${ }^{1 *}$.

\section{RESUMO}

Objetivo: Relatar um caso de amenorréia primária, a investigação ambulatorial desse quadro e discutir alterações genéticas e endocrinológicas envolvidas. Detalhamento de caso: Paciente do sexo feminino, com idade de 16 anos, encaminhada ao ambulatório de endocrinologia ginecológica por ausência de menarca e caracteres sexuais secundários hipodensenvolvidos. Ao exame físico broto mamário presente à esquerda e ausente no lado direito, pêlos axilares e vaginais esparsos, Tanner M1P1. Investigação ambulatorial com dosagens hormonais e imagens ultrassonográficas sugestivas de insuficiência ovariana, hipótese diagnóstica confirmada por cariotipagem com evidência de disgenesia gonadal. Posterior terapêutica com estradiol e seguimento para avaliação da resposta à terapia hormonal e realização de exames complementares. Considerações finais: $O$ pseudo isodicêntrico $X$ reflete uma alteração genética que resulta no quadro de hipogonadismo hipergonadotrófico e, tal condição fisiopatológica explica a amenorréia primária e o não desenvolvimento sexual secundário apresentados pela paciente em questão. Desdobramentos biológicos e psicossociais exigem abordagem terapêutica e seguimento médico pelos possíveis desfechos associados.

Palavras-chave: Amenorreia, Desenvolvimento sexual, Cromossomo pseudo isodicêntrico.

\begin{abstract}
Objective: Report a case of primary amenorrhea, the ambulatory investigation about the condition, and discuss the genetic and endocrinological changes associated. Case details: A 16 years old female patient was referred to the gynecology endocrinological ambulatory due to an absence of menarche and incomplete secondary sex characteristics development. On the physical exam breast bud noted at the left and absent on the right side, sparse axillary and vaginal hair, Tanner M1P1. The laboratory research started with hormonal dosages and ultrasound images suggestive of ovarian insufficiency, diagnosis hipoteses then confirmed by karyotyping wich also showed gonadal dysgenesis. Then it was started estrogen hormone therapy and attendance to evaluate the therapy response and some complementary exames. Final considerations: Hypongonadis hypergonadotrofic as result of genetic changes in the $\mathrm{X}$ cromossome (pseudo isodicentric) were responsible for the primary amenorrhea and the incomplete secondary sex characteristics development. The biological, psychological and social consequences demand a therapeutic approach and a medical follow-up due to the possible clinical ways that this condition can advance.
\end{abstract}

Keywords: Amenorrhea, Sex development, Pseudo isodicentric cromossome.

\section{RESUMEN}

Objetivo: Relatar un caso de amenorrea primaria, la investigación ambulatoria de este cuadro, y discutir alteraciones genéticas y endocrinólogas involucradas. Detalles del caso: Paciente del sexo femenino, con edad de 16 años, referida al ambulatorio de endocrinología ginecológica por ausencia de menarquía y desarrollo sexual secundario incompleto. En el examen físico, se observa una protuberancia mamaria presente a la izquierda y ausente del lado derecho, vellos axilares y vaginales dispersos, Tanner M1P1.

${ }^{1}$ Universidade de Franca (UNIFRAN), Franca - SP.

2 Fundação Santa Casa de Misericórdia de Franca, Franca - SP. *E-mail: ronaldotcastro@hotmail.com 
Investigación ambulatorial con dosis hormonales e imágenes ultrasonográficas indicador de insuficiencia ovárica, hipótesis diagnóstica confirmada por cariotipo con evidencia disgenesia gonadal. Posterior terapéutica con estradiol y seguimiento para evaluación de respuesta a la terapia hormonal y de exámenes complementarios. Consideraciones finales: Alteraciones genéticas del cromosoma X (pseudo isodicéntrico) resultaron en un cuadro de higonadismo hipergonadotrófico, tal condición fisiopatológica explica la amenorrea primaria y el no desarrollo sexual secundario presentados por la paciente en cuestión. Desdoblamientos biológicos y psicosociales exigen un abordaje terapéutico y seguimiento médico por los posibles resultados asociados.

Palabras clave: Amenorrea, Desarrollo sexual, Cromosoma pseudo isodicéntrico.

\section{INTRODUÇÃO}

O termo amenorreia, utilizado comumente na prática médica, refere-se a um estado onde não há a ocorrência da menstruação. Se a menarca (evento que marca a primeira menstruação) ocorreu dentro do padrão normal, mas por alguma condição, não necessariamente patológica, houve uma interrupção da menstruação por no mínimo três ciclos ou por mais de seis meses contínuos, o quadro se encaixa na chamada amenorreia secundária. Enquanto que a amenorreia primária designa um grupo de mulheres que não tiveram a menarca, frequentemente, entre 10 a 14 anos de idade (SAMAL R e HABEEBULLAH S, 2017).

$\mathrm{Na}$ amenorreia primária existe uma subdivisão que auxilia na investigação etiológica de sua causa; um grupo de meninas com 16 anos, com ausência da menarca, mas que apresentam o desenvolvimento de caracteres sexuais secundários e um segundo grupo de meninas com 14 anos, que além da ausência da primeira menstruação, também não apresentam desenvolvimento dos caracteres sexuais secundários (SAMAL R e HABEEBULLAH S, 2017).

Em meninas pertencentes ao primeiro grupo cogita-se o comprometimento em órgãos internos do sistema reprodutor - como vagina, útero, ovários - ou em regiões como hipotálamo e hipófise (MASTER-HUNTER T e HEIMAN DL, 2006). Nesse sentido, a Ultrassonografia (USG) e a Ressonância Magnética (RM) representam exames eficientes para avaliação anatômica interna inicial (RONCHETTI MR, et al., 2013).

As pacientes de 14 anos que não desenvolveram os caracteres sexuais secundários, em um momento inicial, devem ter os níveis séricos de alguns hormônios dosados na tentativa de identificar a fisiologia comprometida, e a partir disso guiar os próximos exames complementares para enfim alcançar um diagnóstico. Os hormônios a serem avaliados são: hormônio estimulante da tireóide (TSH), prolactina (PRL), androgênios, hormônio luteinizante (LH) e hormônio folículo estimulante (FSH) (MASTER-HUNTER T e HEIMAN DL, 2006).

Os hormônios gonadotróficos são representantes do eixo hipotálamo-hipófise-ovariano e assim, valores diminuídos de LH e FSH revelam uma disfunção hipotalâmica ou hipofisária. Nesses casos, o estímulo necessário para o desenvolvimento dos ovários não acontece, e por isso essa condição é denominada hipogonadismo hipogonadotrófico. O foco da investigação é então identificar qual estrutura central está comprometida e, a RM é a ferramenta utilizada para visualização do hipotálamo e da hipófise (SAMAL R e HABEEBULLAH S, 2017).

Por outro lado, os valores aumentados de FSH e LH traduzem uma situação de integridade central; o estímulo para desenvolvimento ovariano existe, mas os ovários, por alguma razão, não se desenvolvem (SAMAL R e HABEEBULLAH S, 2017). Logo, essa é uma condição chamada hipogonadismo hipergonadotrófico e, o cariótipo é o exame complementar solicitado para seguimento do diagnóstico (RONCHETTI MR, et al., 2013).

Anormalidades do cromossomo X - estruturais ou numéricas - representam uma porcentagem significativa entre as causas de amenorreia primária. Uma das irregularidades que podem ser encontradas nesse exame é o mosaicismo, podendo ser, em alguns casos, um indicativo de uma condição chamada disgenesia gonadal que, quando associada a amenorreia e o não desenvolvimento de caracteres sexuais secundários, refere-se a uma falência ovariana precoce (ROSA RFM, et al., 2008). 
Tanto a amenorreia primária quanto o não desenvolvimento de caracteres sexuais secundários são reflexos dessa falência ovariana prematura. Além disso, a insuficiência estrogênica pode resultar em comprometimentos ósseos, e influenciar na estatura final da paciente. Em relação ao tratamento medicamentoso, o objetivo inicial é o desenvolvimento dos caracteres sexuais secundários e a consequente feminização. É interessante ressaltar que devido a diversidade das consequências recomenda-se uma abordagem multidisciplinar que monitora cada aspecto a ser influenciado pela insuficiência ovariana (RONCHETTI MR, et al., 2013).

O objetivo desse trabalho é relatar o processo de investigação ambulatorial de um caso de amenorreia primária por hipogonadismo hipergonadotrófico. As alterações endocrinológicas e genéticas dessa condição são discutidas a partir do quadro clínico, laboratorial e de imagem da paciente. Assim como alguns aspectos da linha de seguimento terapêutico adotado à portadora da disgenesia gonadal.

\section{DETALHAMENTO DO CASO}

No ano de 2020 uma versão do projeto foi submetida ao Comitê de Ética em Pesquisa da Fundação Santa Casa de Misericórdia de Franca que viabilizou o desenvolvimento do estudo de caso a partir do parecer de número 4.145.982. A data de aprovação foi no dia nove de julho de 2020.

HFS, sexo feminino, 16 anos, foi referenciada ao Ambulatório Escola de uma universidade do Estado de São Paulo para especialidade de Ginecologia Endócrina após consulta na área de Ginecologia Geral, por conta de quadro de amenorreia primária. Estava acompanhada da mãe e na entrevista referiu ausência de menarca e do desenvolvimento das mamas. Relatou presença de pelos axilares e pubianos, não se recordando da idade da pubarca. Ao interrogatório complementar negava sexarca, vícios ou uso de medicamentos.

Ao exame físico, notada presença de broto mamário apenas em mama esquerda, apresentava esparsos pêlos axilares e escala de Tanner M1P1. Ao exame ginecológico, notada presença de pequenos lábios atróficos, não evidenciando lesões em hímen. A escala citada é utilizada para acompanhamento evolutivo de mudanças sexuais da puberdade, no sexo feminino os paramêtros avaliados são a idade da paciente, o estágio de desenvolvimento mamário e de pilificação pubiana.

Foram apresentados exames laboratoriais e USG pélvica solicitados anteriormente. Dos laboratoriais, apenas o nível de FSH apresentava-se elevado com valor de 103,46 mUI/L (Valor referência: Fase Folicular Média 3,85 a 8,78 $\mathrm{mUI} / \mathrm{ml}$ ). A USG revelou um útero anteroversofletido com contornos regulares e volume de $3,8 \mathrm{~cm}^{3}$, miométrio homogêneo e endométrio de $1,2 \mathrm{~mm}$ podendo corresponder à útero rudimentar. Como conduta foi solicitado USG de abdome total e novos exames laboratoriais complementares como dosagem de androstenediona, desidroepiandrosterona (DHEAS), testosterona total, estradiol e globulina ligadora de hormônios sexuais (SHBG) e cariótipo.

Ao retorno, no mês seguinte, a paciente negou novas queixas e apresentou o resultado dos exames solicitados sem alterações significativas. Foi realizado vaginometria com comprimento vaginal de $7 \mathrm{~cm}$. Optouse por conduta expectante até resultado de cariótipo.

Dois meses após a última consulta, a paciente retornou sem outras queixas e com o resultado da cariotipagem contendo 46X, psiu idic (X) (q28). Com isso, foi firmado o diagnóstico de amenorreia primária de origem ovariana e como conduta foi prescrito valerato de estradiol na dose 2,0 $\mathrm{mg}$ por 60 dias e posterior reavaliação.

Após sessenta dias em uso de valerato de estradiol 2,0 mg, paciente comparece a consulta para avaliação, foi relatado aumento no tamanho da glândula mamária e aumento da pilificação. Em relatório da radiografia de mãos e punhos para avaliação de idade óssea, realizado anteriormente, foi descrito, segundo o método de Greulich-Pyle para padrão feminino, idade óssea em torno de onze anos. Esse resultado é dado a partir de uma análise comparativa entre a radiografia e imagens de um Atlas que demonstra diferentes graus de maturação óssea das regiões radiografadas. Durante o exame físico avaliação de Tanner se manteve M1P1. Nessa mesma consulta, foi solicitado uma RM de pelve e crânio, e a dose de valerato de estradiol aumentada para $3,0 \mathrm{mg}$. 
Ao exame de RM de crânio, estruturas avaliadas apresentavam padrão estrutural adequado, a impressão diagnóstica do examinador confirmava integridade anatômica de sela túrcica.

Paciente fez uso da última medicação prescrita (valerato de estradiol $3,0 \mathrm{mg}$ ) por um intervalo de 3 meses. Em retorno, após 6 meses desde a última consulta, solicitou a troca da terapia hormonal em uso por anticontraceptivos orais, ao exame físico Tanner M2P2. Prescrito etinilestradiol na dosagem de 0,3 mcg associado a levonorgestrel $0,15 \mathrm{mcg}$, após seis meses de uso paciente apresentou episódio de menarca.

\section{DISCUSSÃO}

A puberdade é um evento de modificações biológicas que culminam na maturidade sexual-reprodutiva e estabelecimento final da estatura. O fator neuroendrócrino desencadeante é a reativação de núcleos hipotalâmicos, onde determinantes genéticos e ambientais influenciam nesse processo (KNUDTSON J, 2016). Os neurônios hipotalâmicos iniciam um formato específico de secreção pulsátil do hormônio liberador de gonadotrofina $(\mathrm{GnRH})$, neurohormônio que age no controle secretório de FSH e LH em gonadotropos hipofisários (SEMBULINGAM K e SEMBULINGAM P, 2016).

Os caracteres sexuais secundários observados no desenvolvimento puberal feminino são resultados, principalmente, da atuação de esteróides sexuais - estrogênios, progesterona e androgênios/testosterona (BREEHL L e CABAN O, 2020). O desenvolvimento das mamas, a menarca e o crescimento do útero e ovário são eventos dependentes de hormônios ovarianos. Enquanto a pilificação axilar e pubiana podem acontecer em situações de hipogonadismo por atuação de andrógenos suprarrenais como no caso descrito, no qual se pôde observar discreta pilificação, apesar do hipogonadismo (SAMAL R e HABEEBULLAH S, 2017). E assim como na literatura, na paciente, não houve o desenvolvimento dos caracteres secundários e útero-ovárico.

A paciente em questão no momento da consulta tinha 16 anos, geralmente a primeira manifestação da puberdade feminina é o aparecimento do broto mamário (telarca), e acontece em média com 9,7 anos (+/- 3 anos). O desenvolvimento de caracteres sexuais secundários é avaliado por meio de um sistema desenvolvido por Tanner, que utilizou como parâmetro de avaliação a relação entre as idades de aparecimento com a pilificação pubiana $(P)$ e, no caso das meninas, também o desenvolvimento mamário (M) (Quadro 1) (KNUDTSON J, 2016; MENESES C, et al., 2008). No caso relatado o resultado da aplicação de Tanner foi M1P1, porém esperava-se que ao exame físico ela apresentasse M5P5.

Quadro 1 - Escala de Tanner - Desenvolvimento puberal feminino.

\begin{tabular}{|c|l|}
\hline \multicolumn{2}{|c|}{ MAMAS } \\
\hline M1 & Mama infantil. \\
\hline M2 (8-13 anos) & $\begin{array}{l}\text { Broto mamário, pequena elevação de mama e da papila com aumento do diâmetro da } \\
\text { aréola. }\end{array}$ \\
\hline M3 (10-14 anos) & Aumento da mama e da aréola, mas sem separação dos contornos dessas estruturas. \\
\hline M4 (11-15 anos) & $\begin{array}{l}\text { Crescimento e projeção da aréola e da papila, com a formação de uma elevação } \\
\text { acima do corpo mamário. }\end{array}$ \\
\hline M5 (13-18 anos) & $\begin{array}{l}\text { Fase adulta, com saliência somente nas papilas. Aréola retorna para contorno geral } \\
\text { da mama. }\end{array}$ \\
\hline \multicolumn{2}{|c|}{ PELOS } \\
\hline P2 (9-14 anos) & $\begin{array}{l}\text { Crescimento esparso de pelos finos, levemente pigmentados, lisos ou pouco } \\
\text { encaracolados ao longo dos grandes lábios. }\end{array}$ \\
\hline P3 (10-14,5 anos) & Pelos mais escuros e encaracolados, alcançam a sínfise púbica. \\
\hline P4 (11-15 anos) & Pelos do tipo adulto, sem alcançar a superfície interna das coxas. \\
\hline P5 (12-16,5 anos) & $\begin{array}{l}\text { Pelos do tipo adulto em quantidade e características alcançam a superfície interna } \\
\text { das coxas. }\end{array}$ \\
\hline
\end{tabular}

Fonte: Cecchi AO, et al., 2021; baseado em Knudtson J, 2016.

A paciente foi admitida no ambulatório já com 16 anos de idade, logo não houve um acompanhamento progressivo de seu crescimento estatural. Entretanto foi solicitado uma radiografia de mãos e punhos, isso porque normalmente a maturação sexual é proporcional à maturação óssea e não à idade cronológica. $\mathrm{A}$ 
menarca (geralmente na fase M4 de Tanner) coincide com a desaceleração do crescimento estatural, evento que se inicia em M2, não tem influência direta de hormônios ovarianos, mas em situações de hipogonadismo pode estar prejudicado pelo consequente atraso puberal (DUARTE MFS, 1993; KNUDTSON J, 2016). O resultado do exame solicitado (com referência aproximada de 11 anos) demonstra retardo da maturação óssea, cenário condizente com a patologia estudada.

Ao avaliar os exames laboratoriais solicitados à paciente, o valor do FSH apresentava-se elevado pela ausência de retroalimentação negativa na secreção de $\mathrm{GnRH}$ - o que mantém o estímulo aos receptores hipofisários aumentado e impede a modulação dos receptores gonadotrofos.

Em condições adequadas o conjunto neuronal hipotalâmico responsável pela secreção pulsátil de GnRH pode ter atuação esporádica ou regulada. A influência adrenérgica positiva acontece por ação direta da noradrenalina, a negativa envolve ácidos gama aminobutírico $A$ e $B$ (GABA). A regulação pode também ser pela dopamina, neurotransmissor responsável pela inibição da síntese e liberação de GnRH (FEBRASGO, 2015).

Esteróides sexuais como progesterona e andrógenos são inibitórios dessa secreção; a retroalimentação estrogênica no hipotálamo depende da concentração plasmática e do tempo de atuação. É positiva quando níveis elevados são sustentados por um período de 50 horas, diferente disso o hormônio diminui a ação estimuladora do neurohormônio kisspeptina (TRISOTTO ALA, 2020; FEBRASGO, 2015).

Nos gonadotropos hipofisários existem receptores específicos de $\mathrm{GnRH}$, cujo estímulo pulsátil associa-se à liberação das gonadotrofinas. A inibina, esteróides sexuais, entre outros, também podem modular a resposta desses receptores ao $\mathrm{GnRH}$, justificando que durante o ciclo menstrual, em que as concentrações de esteróides sexuais não são fixas, os níveis de LH e FSH também são variáveis (FEBRASGO, 2015). A confirmação da integridade do eixo hipotálamo-hipófise foi confirmada pelas dosagens hormonais e RM da sela túrcica durante o seguimento ambulatorial.

Apesar da integridade dos centros superiores de regulação hormonal, e consequente secreção de LH e $\mathrm{FSH}$, esses neurohormônios parecem não exercer influência sob os tecidos ovarianos da paciente. Dessa maneira, não haverá produção tanto de estrógeno quanto progesterona, o que resultou no quadro de hipogonadismo. Além disso, a ausência de estrogênio impede a fase proliferativa uterina, e consequentemente não haverá a descamação, ou seja, a menstruação; o que ocorreu com a paciente do caso

A partir da puberdade, a fisiologia ovariana é regulada pelas gonadotrofinas onde o crescimento folicular, que se inicia em período intrauterino, tem sua fase final controlada pelos pulsos de LH e FSH. A função endócrina, produção de esteróides sexuais, se inicia pela ação específica do LH em receptores localizados nas células TECA que promove a conversão de colesterol em testosterona e androstenediona. Esses andrógenos se difundem às células da granulosa e são convertidos, sob comando do FSH e por meio da enzima aromatase, em estrógenos. A progesterona e o estradiol são formados quando as células da granulosa estão em estágio de corpo lúteo (FEBRASGO, 2015; THIYAGARAJAN DK, et al., 2019).

A menstruação depende da ação desses hormônios. Em conjunto com fatores de crescimento, o estrogênio promove no endométrio a proliferação de vasos e elevação da atividade mitótica, o que resulta em aumento do espessamento endometrial que após a ovulação é sustentado pela progesterona secretada pelo corpo lúteo. Sem a fecundação, esses hormônios têm seus níveis diminuídos e com isso a desagregação endometrial progressiva. O fluxo menstrual é consequência desse processo (THIYAGARAJAN DK, et al., 2019).

Em situação de hipogonadismo, como apresentado pela paciente, a reposição de estrogênios conjugados objetiva cumprirem o papel, mais especificamente, do estrogênio fisiológico. A ausência desse hormônio, somada à disfunção no desenvolvimento sexual secundário, compromete a sexualidade, associa-se a sintomas urinários, aumenta o risco de doenças coronarianas, e acelera a velocidade de perda óssea, entre outros prejuízos negativos (SWEE DS, et al., 2019).

O resultado da citogenética revelou alterações no cromossomo $X$, principal envolvido nas disgenesias gonadais relatadas na literatura (ROSA RFM, et al., 2008). A clínica depende da posição da quebra, presença 
ou não de mosaico e padrão de inatividade (PEREZA N, et al., 2011). Nesse caso é um pseudo isodicêntrico do $X$ (q28) sem constatação de mosaicismo. Esse dado contrasta com a literatura onde tem-se a que a maioria do $(X p)$ e $(X q)$ ocorrem como mosaico, associados com uma linhagem 45,X, e não como uma linhagem apenas contento isodicêntricos do cromossomo X (HOWELL RT, et al., 1976; MELARAGNO MI. et al.,1993). Especificamente, pacientes não mosaicos 46,X,idic $(X q)$ são raros.

Em 50\% dos pacientes com Síndrome de Turner, média de idade do diagnóstico de 12 anos, o cariótipo mais frequente é 45,X (monossomia total do cromossomo X) em contrapartida da monossomia parcial desse mesmo cromossomo. Dentro desse último, vários cariótipos podem estar associados com a presença de dicêntricos e isodicêntricos (SCOTT S, et al., 2010; MARQUI ABT, 2015). No presente caso, a paciente apresenta um cariótipo com isodicêntrico do cromossomo X em 100\% das células analisadas (WONG MSF e LAM STS, 2005).

Conclui-se que os possíveis desdobramentos fisiopatológicos que envolvem principalmente o hipoestrogenismo impõe a necessidade de seguimento clínico. A paciente em questão foi acompanhada de perto, com consultas frequentes e exames complementares específicos. A terapêutica foi iniciada precocemente e os resultados foram avaliados por meio, principalmente, da anamnese e exame físico nos atendimentos seguintes. Ajustes medicamentosos necessários tanto para evolução clínica favorável quanto para prevenção de complicações do quadro foram realizados, levando em consideração também os desejos da paciente, fator essencial para adesão ao tratamento.

\section{REFERÊNCIAS}

1. BRASIL. Manual de Orientação Ginecologia Endócrina. 2010 . Disponível em: http://professor.pucgoias.edu.br/SiteDocente/admin/arquivosUpload/13162/material/GINECOLOGIA\%20END\%C3\%9 3CRINA\%20-\%20FEBRASGO\%202010.pdf. Acesso em: 4 de dezembro de 2019.

2. BREEHL L, CABAN O. Physiology, Puberty. Online: StatPearls Publishing LLC, 2020. Disponível em: https://www.ncbi.nlm.nih.gov/books/NBK534827/. Acesso em: 13 de janeiro de 2021.

3. DUARTE MFS. Maturação física: uma revisão da literatura, com especial atenção à criança brasileira. Caderno Saúde Pública, 1993; 9(1): 71-84.

4. HOWELL RT, et al. Dicentric X isochromosomes in man. Journal of Medical Genetics, 1976; 13(6): 496-500.

5. KNUDTSON J, MCLAUGHLIN JE. Endocrinologia Reprodutiva Feminina. Manual MSD, 2016. Disponível em: https://www.msdmanuals.com/pt-br/profissional/ginecologia-e-obstetr\%C3\%ADcia/endocrinologia-reprodutivafeminina/endocrinologia-reprodutiva-feminina. Acesso em: 20 de janeiro de 2021.

6. MACEDO DB, et al. Avanços na etiologia, no diagnóstico e no tratamento da puberdade precoce central. Arquivos Brasileiros de Endocrinologia e Metabologia, 2014; 58(2): 108-117.

7. MARQUI ABT. Síndrome de Turner e polimorfismo genético: uma revisão sistemática. Revista Paulista de Pediatria. 2015; 33(3): 363-370.

8. MASTER-HUNTER T, HEIMAN DL. Amenorrhea: Evaluation and Treatment. American Family Physician. 2006; 73(8): 1374-1382.

9. MELARAGNO $\mathrm{Ml}$, et al. Isodicentric $X$ chromosome and mosaicism: Report on two cases of $45, X / 46, X, \operatorname{idic}(X q) / 47, X, \operatorname{idic}(X q)$, idic(Xq) and review of the literature. American Journal of Medical Genetics, 1993; 47(3): 357-359.

10. MENESES C, et al. Estagiamento de Tanner: um estudo de confiabilidade entre o referido e o observado. Revista Adolescência e Saúde, 2008; 5(3).

11. PEREZA N, et al. Izodicentrični X kromosom i složeni mozaicizam 45, $X / 46, X$,idic(X)(q28)/46, XX u bolesnice sa sekundarnom amenorejom, visokim rastom i pretilošću. Medicina Fluminensis, 2011; 47(1): 107-114.

12. RONCHETTI MR, et al. Amenorréia primária: fluxograma de investigação. Acta Médica (Porto Alegre), 34(5).

13. ROSA RFM, et al. Amenorréia e anormalidades do cromossomo X. Revista Brasileira de Ginecologia e Obstetrícia, 2008; 30(10).

14. SAMAL R, HABEEBULLAH S. Primary amenorrhea: a clinical review. International Journal of Reproduction, Contraception, Obstetrics and Gynecology, 2017; 6(11): 4748.

15. SCOTT S, et al. Large Inverted repeats within Xp11.2 are present at the breakpoints of isodicentric $X$ chromosomes in Turner syndrome. Human Molecular Genetics, 2010; 19(17): 3383-3393.

16. SEMBULINGAM K, SEMBULINGAM P. Menstrual Cycle. Essentials of Medical Physiology, $2012 ; 482-482$.

17. SWEE DS, et al. Estrogen Replacement in Young Hypogonadal Women-Transferrable Lessons From the Literature Related to the Care of Young Women With Premature Ovarian Failure and Transgender Women. Frontiers in Endocrinology, 2019; 10: 685.

18. THIYAGARAJAN DK, et al. Physiology, Menstrual Cycle. Online: StatPearls Publishing LLC, 2020. Disponível em: https://www.ncbi.nlm.nih.gov/books/NBK500020/. Acesso em: 10 de janeiro de 2021.

19. TRISOTTO ALA. Puberdade Precoce: Avaliação do desenvolvimento de crianças tratadas com análogos de GnRH. Trabalho de Conclusão de Curso (Graduação em Farmácia) - Centro de Ciências da Saúde. Universidade Federal de Santa Catarina, 2020.

20. WONG MSF, LAM STS. Cytogenetic analysis of patients with primary and secondary amenorrhoea in Hong Kong: retrospective study. Hong Kong Medical Journal, 2005; 11(4). 